\title{
Practical suggestions for better crystal structures
}

\author{
Peter Müller* \\ Department of Chemistry, Massachusetts Institute of Technology, Cambridge, MA, USA
}

(Received 15 September 2008; final version received 9 October 2008)

\begin{abstract}
X-ray crystallography is one of the most popular analytical methods, and with the advent of area detectors in the 1990s single crystal X-ray structure determination has become commonplace. Initially, the method was reserved for the expert, but hard- and software improvements of the last couple of decades have enabled scientists who are not formally trained in crystallography to determine crystal structures as well. This has led to an explosion of the number of crystal structures and, unfortunately, also of the number of incorrect structures submitted to scientific journals. It is evident that (semi)automated structure determination works only for routine structures. In more complex cases, such as structures with disorders, pseudo-symmetry or twinning, crystallographic knowledge, refinement skills and experience are still vital for obtaining high-quality, publication-grade crystal structures. This article is meant to offer a few suggestions to scientists who are using crystallography as part of their research, as an ancillary-science so to speak, hoping to help improve the quality of their crystal structures.
\end{abstract}

Keywords: structure refinement; crystal growth; data collection strategy; structure solution; restraints; constraints; disorder

\section{Contents}

2. Pre-refinement strategies $\quad 58$

2.1. Crystal growth and crystal handling 59

2.2. Choosing a crystal, mounting and diffractometer alignment 60

2.3. Data collection strategy 61

2.4. Absorption correction $\quad 62$

2.5. Structure solution $\quad 62$

3. Structure refinement 63

3.1. Parameters $\quad 63$

3.2. Constraints $\quad 64$

3.3. Restraints 65

3.4. General strategies $\quad 69$

*Email: pmueller@mit.edu 
4. Examples 70

4.1. Splitting coordinates step-by-step $\quad 71$

4.2. Disordered $\mathrm{CF}_{3}$ groups $\quad 72$

4.3. Too short $\mathrm{N}-\mathrm{N}$ bond in dinitrogen $\quad 75$

4.4. Overlooked inversion centre 76

$\begin{array}{ll}\text { 5. Summary } & 78\end{array}$

$\begin{array}{ll}\text { References } & 79\end{array}$

\section{Introduction}

In some respects the training to become a crystallographer is similar to that of a Jedi Knight, as the practical knowledge usually only goes from the master's mouth to the apprentice's ear. Therefore, it can be difficult for the autodidact to become adept without a local guru's help. Very few textbooks on practical Crystal Structure Refinement $(1,2)$ and only a small number of articles with truly practical refinement advice (3-7) offer help to the less experienced crystallographer, even though the large number of erroneous structures submitted to scientific journals every year clearly indicates the need of education and practical training.

The determination of a crystal structure consists of several steps all of which pose their individual challenges: After crystal growth, unit cell determination, data collection, data reduction, space group determination and structure solution, the crystallographer has obtained atomic coordinates for some or all non-hydrogen atoms. Frequently the atom types assigned to some of the initial coordinates are incorrect and many details of the structure, such as groups of lighter atoms, disorders, hydrogen positions, etc., may still be missing. The way from the first solution to the final accurate and publishable model is called refinement and depending on the structure, this can be a short highway to happiness or a rough and rugged road through pain and sorrow.

This article is meant to give some practical advice, mostly about structure refinement without, however, focusing on any specific software package. It is aimed at scientists using crystal structure determination as part of their research, and was written hoping to help improve the quality of their crystal structures. For refinement advice related to specific software packages, the reader may turn to the above mentioned book by Müller et al. (1), which is based on the program SHELXL (6) and offers a large number of workshop-like practical refinement examples, or to the software documentation directly.

\section{Pre-refinement strategies}

In general, there are two ways of getting around in the world: learning how to deal with problems and staying out of trouble in the first place. While the first is a necessity of life (and crystallography), the second one is more elegant and usually less time consuming (albeit less exciting). Therefore, before we discuss refinement techniques for difficult structures, let us spend some time optimizing data quality first. Taking care of every step and aspect of the whole diffraction experiment is equivalent to staying out of trouble. According to the fundamental equation 'Garbage in = Garbage out', it is much easier to refine a structure based on good than on bad data and time invested into improving data quality is returned with interest in the refinement stage. 

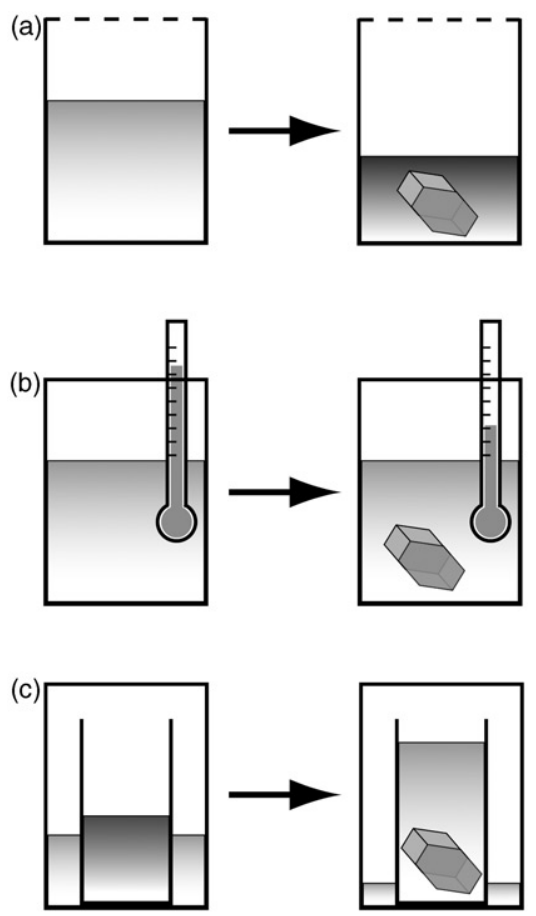

Figure 1. Schematics of three popular methods of crystal growth. (a) Slow evaporation of the solvent, (b) slow cooling of the solution and (c) gas-phase diffusion of a precipitant into a solution. Method (c) usually gives the best crystals of the three methods shown.

\subsection{Crystal growth and crystal handling}

Theoretically, crystallization starts when the concentration of a compound in a solvent is higher than the solubility product of this compound. Generally, however, crystallization is kinetically hindered and crystals grow only from supersaturated solutions. There are several ways to achieve this metastable state of supersaturation.

The easiest approach is to increase the concentration by evaporation of the solvent until crystallization sets in, for example by not closing the lid of the tube or flask very tightly and simply wait [Figure 1(a)]. ${ }^{1}$ A potential problem with this approach is that with the concentration the crowding in the solution increases, which can lead to too much nucleation and, in turn, to an increased number of packing defects in the crystal as well as to many too small crystals instead of a few bigger ones.

Another way of obtaining a supersaturated solution is making use of the fact that many compounds are better soluble in hot solvents than in cold ones. An almost saturated hot solution is likely to yield crystals at room temperature [Figure 1(b)]. However, crystals that start growing at higher temperature are frequently twinned or show static disorder, as the sometimes small energy differences between conformations become insignificant at higher temperature, which leads to disorders and packing defects in the resulting crystals.

\footnotetext{
${ }^{1}$ Many crystals are obtained from NMR tubes. NMR tubes are usually sealed with a little colourful plug, which is not overly tight. When left in the fridge for several weeks or months, the solvent left slowly evaporates from the NMR tube, the solution becomes first saturated, then supersaturated and crystals grow.
} 
Usually, the best technique to grow high-quality crystals is the use of binary solvent systems. One needs two liquids that mix well, and the compound to be crystallized should be soluble in only one of them. The liquid in which the compound is soluble is called the solvent, the other liquid the precipitant. As the compound is less soluble in a mixture of the two liquids, one can grow crystals by slowly mixing a not too concentrated solution of the target compound with the precipitant. The slow mixing can happen as liquid-liquid diffusion, gas-phase diffusion [shown in Figure 1(c)] or via a membrane (dialysis). The striking advantage of these binary solvent systems is the fact that the supersaturation is reached while the concentration and with it the molecular crowding actually decreases. It means that crystals can be grown slowly (the diffusion rate can be regulated via the temperature) and usually are much better and more regular in shape than crystals grown from simple evaporation or cool-down experiments.

Once one has obtained a batch of beautiful crystals, it is important to disturb them as little as possible before their time comes to go to the diffractometer. That means the environmental conditions should not be changed unnecessarily and trips to the microscope to admire the crystals should be kept to a minimum. Most importantly, crystals should not be separated from the mother liquor in which they grew before it is time to mount them. If solvent molecules have co-crystallized with the target compound, drying the crystals will destroy them, as, over time, solvent molecules will evaporate from the crystal lattice, leaving open voids. The result is usually a complete collapse of the crystal lattice - the crystal turns into a powder - or at least severe damage to the crystal, rendering it unusable for a diffraction experiment. Even if a crystal contains no solvent molecules, it is advisable to keep it wet, as exposure to air can damage a fragile crystal significantly.

\subsection{Choosing a crystal, mounting and diffractometer alignment}

It is worthwhile spending time on finding the best crystal of the batch. Usually, a good crystal shows well-defined, sharp crystal faces, is shaped regularly (no plate or needle) and is clear rather than opaque. It is recommended to choose a crystal that is smaller than the diameter of the primary beam to make sure that the amount of irradiated matter remains constant regardless of crystal orientation and hence throughout the entire data collection.

Every crystallographic laboratory should be equipped with a polarizing microscope, as birefringence helps to distinguish single crystals from macroscopically twinned ones. If all crystals appear to be inter-grown and stuck together (or if all crystals are larger than the primary beam), there is no shame in using a scalpel or razor blade to surgically isolate a good specimen or cut it to size.

With an area detector, now standard on most diffractometers, the quality of a crystal can also be checked by a number of quickly collected diffraction patterns. It should become a habit to take a number of diffraction images in at least three or four different crystal orientations before a data collection is started.

Finally, it is imperative to keep the instrument well maintained and correctly aligned. The zero points of all circles and the detector parameters should be checked regularly and updated when necessary. For CCD detectors the dark current ${ }^{2}$ correction files need to be

\footnotetext{
${ }^{2}$ The dark current is the noise produced by CCD chip and readout electronics in the absence of Xray photons (i.e. in the dark). The dark current depends mostly on exposure time and chip temperature, but can fluctuate for other reasons as well.
} 
kept up-to-date. It is good practice for a busy facility to collect a set of dark current files for every commonly used exposure time (say 5, 10, 20 and 30 s) in weekly intervals. At least four times per year a known test structure should be determined from scratch, ideally always from the same crystal. Comparison of the results (detector errors from unit cell determination, merging $R$-values and $R$-values of the refinement) with those of the previous determinations of the same structure allows for assessment of the condition of the instrumentation and any problem with the test structure should be investigated. The test structure should also be determined whenever doubts about the instrumentation arise, for example after diffractometer repairs or when it suddenly becomes difficult to determine unit cells or when all bond lengths are determined systematically too long or short.

\subsection{Data collection strategy}

The data collection strategy should always match the problem and the instrumentation. An excellent introduction to the topic is given by Dauter (8). In general, observed intensities are weaker at higher resolution and almost no crystal diffracts to the theoretical diffraction limit of $d_{\max }=\lambda / 2$. Some care must be taken in the determination of the effective maximum resolution of a dataset. There are at least five qualifiers describing the quality of a dataset: maximum resolution, completeness, multiplicity of observations (MoO, sometimes called redundancy), ${ }^{3}$ the average value of measured intensity divided by the estimated noise $\langle I / \sigma\rangle$ and a variety of merging residual values. The four latter qualifiers are usually given as a function of resolution, and most scientists give a pair of values for each qualifier: the average value for the complete dataset and the value for only the highest resolution shell.

A good dataset must be complete (in most cases $99 \%$ or even $100 \%$ completeness can and should be obtained), and for small molecule structures, the International Union of Crystallography (IUCr) requires a good dataset to extend to at least $0.84 \AA$ resolution. A reasonable value for $\mathrm{MoO}$ should be above five to seven for area detector data, but double digit MoO-values are preferable. The value for $I / \sigma$ should be as high as possible (at least 8-10 for the whole dataset), while the lower the merging $R$-factors are the better (most small molecule datasets should show $R_{\text {int }}$ values of below $10 \%$ for the whole resolution range).

As pointed out before $(10,11)$, weak data contain important information and must not be excluded from the dataset (this is, by the way, the most important argument to refine against $F^{2}$ rather than $F$ ). However, there is no reason to use data from highresolution shells when they are all very weak, since these 'reflections' are in fact noise and contain no usable information. There is no generally accepted limit for any of the above mentioned qualifiers, but many crystallographers agree that data with values of $\langle I / \sigma\rangle \leq 2.0$ and/or $R_{\text {int }} \geq 0.45$ throughout the entire resolution shell are to be considered as noise. In practice, there may be more factors to be taken into account and, as always, experience helps.

\footnotetext{
${ }^{36}$ This term was defined at the SHELX workshop in Göttingen in September 2003 to distinguish the $\mathrm{MoO}$ from redundancy, or multiplicity, with which the $\mathrm{MoO}$ has been frequently confused in the past. In contrast to redundancy, which is repeated recording of the same reflection obtained from the same crystal orientation (performing scans that rotate the crystal by more than $360^{\circ}$ ), $\mathrm{MoO}$, sometimes also referred to as "true redundancy", describes multiple measurements of the same (or a symmetry equivalent) reflection obtained from different crystal orientations (i.e. measured at different $\psi$-angles)'. Quoted from Müller et al. (9).
} 
If at all possible, all diffraction data should be collected at low temperature $(100 \mathrm{~K}$ is a good standard, but lower is even better). At low temperature atomic movement is reduced. This increases the observable resolution as well as the overall quality and $I / \sigma$ of the data. As a direct consequence of reduced atomic motion, low-temperature structures are less affected by disorders. Even when a phase transition makes it necessary to collect data near or at room temperature, it is still advantageous to use a low-temperature device. This ensures that the temperature is well defined and remains stable throughout the entire data collection. In addition, the nitrogen stream will protect chemically unstable compounds to some extent. When packing studies are performed, it may be interesting to determine a crystal structure at more than one temperature, as sometimes the packing changes as a function of temperature, and this change is not always accompanied by noticeable cracking of the crystal.

\subsection{Absorption correction}

The single most important statement about absorption correction is that one should always perform one. Even light-atom crystals show some absorption effects and modern semi-empirical methods [e.g. SADABS (12)] are not only highly effective, they also correct for a number of additional effects, such as inconsistencies in primary beam intensity, radiation damage or problems arising from crystals that are larger than the primary beam. A possible control for determining whether a semi-empirical absorption correction was successful can be to check that the values for maximum and minimum transmission $\left(T_{\max }\right.$ and $T_{\min }$ ) estimated by the absorption correction program are reasonable. Frequently, however, the ratio of $T_{\max }$ to $T_{\min }$ is more reliable than their absolute values obtained from the absorption correction software. It should be pointed out that semi-empirical methods are today usually based on equivalent reflections and only work well when the multiplicity of observations is above four to six. Numerical absorption correction (also known as face indexing) is an excellent addition to semi-empirical methods. Experience shows, however, that numerical absorption correction alone is usually inferior to semi-empirical correction alone, which leads to the recommendation to use face indexing only in addition to and not in place of semi-empirical methods.

\subsection{Structure solution}

Most small-molecule crystal structures are solved by direct methods, for example with a program like SHELXS (13) or SIR (14), which are most commonly used as black boxes without any user interaction. In most cases this approach is highly successful, however for the few cases where it is not, here are a few recommendations towards structure solution.

When direct methods fail, Patterson methods may work satisfactorily. Especially with a few satisfactorily heavier atoms in the molecule, the Patterson map can be a very effective starting point for structure solution. Patterson solutions usually contain only a substructure while direct methods frequently give the positions of (almost) all nonhydrogen atoms, however Patterson methods are very fast and the remainder of the atoms is generally revealed in the difference Fourier synthesis after a few refinement cycles.

If a triclinic structure cannot be solved in space group $P \overline{1}$, it is usually solved easily in $P 1$. This is not supposed to mean that $P 1$ is the correct space group, in by far most cases 
$P \overline{1}$ is correct, it only means that direct methods work better in non-centrosymmetric space groups. ${ }^{4}$ Of course, the solution in $P 1$ must then be reduced to $P \overline{1}$ (find the inversion centre, shift the origin accordingly, delete symmetry equivalent atoms and change the symmetry instructions in your input file for the refinement program), but this is usually simple. One such example will be given below (Section 4.4). To a somewhat lesser extent, the same is true for other space groups and in some particularly difficult situations it can be necessary to solve a structure in $P 1$ even though the correct space group may be, say, orthorhombic or tetragonal.

Some structures that cannot be solved with conventional direct methods are solved readily with programs based on dual space algorithms, as implemented in, for example, the programs Shake-and-Bake (15) or SHELXD (6). This is the case in particular for larger light-atom structures, especially when only low-resolution data are available. Classical direct methods stop working around 1.0-1.1 $\AA$, while sometimes dual-space methods can solve structures based on data extending to only $1.5 \AA$.

\section{Structure refinement}

Refinement is the process of iterative alteration of the molecular model with the goal to maximize its compliance with the diffraction data. Following the advice given in the prerefinement strategies section above will result in many good datasets, where refinement is straightforward and fast, and frequently routine methods are sufficient to yield a publishable model within 15-20 min. Sometimes, however, a structure will be difficult in spite of optimal crystal choice and data collection practice, and the refinement of such a structure can take days or weeks. The following pages will present a few techniques that should make life easier when dealing with challenging structures.

In order to successfully refine a difficult structure, it is important to understand what happens during the refinement and to become familiar with the terminology. Reading the excellent articles by Watkin (7) and Sheldrick (6) and references therein is highly recommended. Here are, in brevity, a few fundamentals.

\subsection{Parameters}

A parameter is any quantity that can be refined or adjusted during the process of structure determination. For every atom in the model that is located on a general position in the unit cell, there are three atomic coordinates and six anisotropic displacement parameters to be refined (only one displacement parameter for isotropic atoms). In addition there is one overall scale factor per structure and possibly several additional scale factors, like twin ratios in the refinement of twinned structures, the Flack- $x$ parameter (16) for noncentrosymmetric structures, one parameter for extinction, etc. Some refinement programs like SHELXL (6) allow for additional variables to be refined independently, which can be assigned to various parameters like occupancy factors or interatomic distances.

\footnotetext{
${ }^{4}$ The reason for this is not entirely clear to me, but it appears likely to be related to the fact that in centrosymmetric space groups only two phase angles are possible $\left(0^{\circ}\right.$ and $\left.180^{\circ}\right)$, while in noncentrosymmetric space groups all phase angles are allowed. A phase can be off 'a little bit' in $P 1$, while in $P \overline{1}$ it is either dead-on or completely wrong. That means that in $P 1$ all phases determined approximately right (say within a phase error of $30^{\circ}$ or so) help in constructing an interpretable electron density map, while in $P \overline{1}$ only perfectly correct phases contribute constructively.
} 
Besides these, there is a second group of parameters, which also have significant influence on the structure, and which are usually not refined in the least-squares process but set or adjusted by the crystallographer. These are the atom types and site occupancy factors (sof). The atom types assigned to atomic coordinates determine which atomic scattering factors are used in the Fourier transformation. An incorrectly assigned atom type can cause several kinds of problems, such as making the refined parameters related to this atom adjust to incorrect, and sometimes even meaningless values in an attempt to compensate for the wrong atom type. The sof is most commonly fixed to unity, which means that the atom site is fully occupied (in other words, the atom is present at that site in every unit cell). For atoms on special positions, the sof must be reduced to reflect the multiplicity of the symmetry operator in question ( 0.5 for mirror planes, 0.25 for four-fold axes, etc.), thus ensuring that the asymmetric unit contains only the crystallographicallyindependent portion of the atom. For atoms disordered over two sites in the unit cell, the ratio of the two sofs can be refined, but generally their sum is still fixed to one.

It can be concluded from the above that the number of parameters to be refined depends mostly on the number of crystallographically-independent non-hydrogen atoms and can be assumed to be roughly 10 times the number of atoms in the asymmetric unit for an anisotropic model. A stable and reliable refinement requires a minimum number of observations per refined parameter, and the IUCr community agreed practice currently recommends a minimum data-to-parameter ratio of eight for non-centrosymmetric structures and 10 for centrosymmetric structures. This corresponds to a resolution of about $0.84 \AA$ or a $2 \Theta_{\max }$ of $50^{\circ}$ for $\mathrm{Mo-K} \alpha$ radiation and $134^{\circ}$ for $\mathrm{Cu}-\mathrm{K} \alpha$, respectively. ${ }^{5}$ For many small molecule samples it is not difficult to collect data to $0.75 \AA$ or better, but sometimes a crystal does not diffract well enough. In such cases constraints and restraints can help to indirectly improve the data-toparameter ratio. Excellent descriptions of the use of constraints and restraints in crystal structure refinements have been given by Watkin $(5,7)$.

\subsection{Constraints}

Constraints are equations rigidly relating two or more parameters or assigning fixed numerical values to certain parameters, hence reducing the number of independent parameters to be refined. As mentioned above, site occupancy factors and atom types are usually not refined, but fixed values are assigned to them. This constitutes a constraint.

For atoms on special positions, the sof has to be reduced to reflect the multiplicity of the special position (as explained above) and the shape of the anisotropic displacement ellipsoid must be constrained to make it obey the geometry of the special position in question. In addition it is important to keep the atom fixed on the special position. Take, for example, an atom on a twofold axis coinciding with the $y$ axis. The sof of this atom must be reduced to 0.5 (50\% occupancy), which corresponds to half an atom in each of two adjacent asymmetric units. In addition its anisotropic displacement ellipsoid must be symmetrical with respect to that rotation, which means that the elements $U^{12}$ and $U^{23}$ of the anisotropic displacement tensor must both be zero. In order to fix the atom to stay on the special position, the values for the $x$ and $z$ coordinates of this atom are set to zero.

\footnotetext{
${ }^{5}$ This is assuming that all symmetry-equivalent reflections are merged (except Friedel pairs for noncentrosymmetric structures) and not treated as independent for the calculation of the data-toparameter ratio.
} 
Every one of these restrictions for atoms on special positions is a constraint. Usually all special position constraints are generated automatically by the refinement program, but it is still good to know that such constraints exist and that they reduce the number of independent parameters. More relevant for practical structure refinement are rigid group constraints and identity constraints, which can both be used to cope with challenging refinement situations.

A rigid group is a number of atoms in a given spatial arrangement, for example the six carbon atoms of a benzene molecule forming a perfect hexagon, or seven atoms forming a $\mathrm{PF}_{6}$ octahedron. Rigid groups are allowed to move about the unit cell during refinement, while the overall shape, and usually also the size of the group, does not change. In some cases of heavily disordered groups, in which the individual atomic sites cannot be resolved, but the geometry of the group is known (e.g. a perchlorate ion or a benzene solvent molecule), it can be useful to refine the six parameters of a rigid group (three translational and three rotational parameters) instead of the $3 N$ required parameters for the individual atoms. In addition to the six parameters mentioned, a seventh one can be refined as a bond-length scaling factor to allow the rigid group to 'breathe'. This scaling factor allows the distances among the atoms in the rigid group to be refined, while retaining the overall geometry.

Another common example for an application of rigid group constraints are hydrogen atoms that are refined using a riding model. Hydrogen atoms are frequently placed on geometrically calculated positions and then constraints are applied to the $\mathrm{X}-\mathrm{H}$ bond lengths and $\mathrm{H}-\mathrm{X}-\mathrm{H}$ or $\mathrm{H}-\mathrm{X}-\mathrm{Y}$ angles, setting them to certain values. If the atom carrying the hydrogen moves, the hydrogen atoms move with it (like a rider moves with the horse), keeping the hydrogen bond lengths and angles constant. These constraints are a variation of rigid group constraints, treating the hydrogen atoms bound to a non-hydrogen atom as a rigid group, where the parameters of translation, and in most cases also of rotation, are not refined but derived from the coordinates and geometry of the non-hydrogen atom. This is why adding hydrogen atoms to a model frequently does not increase the number of parameters.

Identity constraints are commonly used on coordinates and anisotropic displacement parameters. When, for example in zeolites or minerals, two different atom types share one crystallographic site in a structure, it can be advisable to use a constraint to force them to do so exactly. In most of such cases, it is also sensible to constrain the anisotropic displacement parameters of those atoms to be identical. Sometimes, in difficult disorders, it can be helpful to constrain pairs of atoms to display the same displacement if molecular geometry warrants it. An example for this situation is given below (Section 4.2).

In principle it is also possible to constrain certain bonds and angles to be identical. However outside of rigid groups, similarity restraints are usually better suited than constraints to stabilize the geometry of molecules. Owing to the mathematical rigidity of constraints, more damage can be done with incorrectly used constraints than with inappropriate restraints and whenever a refinement problem can be solved by means of restraints, this approach should be preferred over the use of constraints.

\subsection{Restraints}

In the absence of restraints, the only data the structural model is refined against are the measured intensities in the form of squared structure factors. Restraints offer a way of 
including additional information one may have about a molecule, for example that aromatic systems tend to be flat or that the three methyl groups in a tert-butyl group are approximately equivalent. Mathematically, restraints are treated as additional experimental observations, thus increasing the number of data to refine against. During structure refinement a Fourier transformation is used to calculate a complete set of structure factors from the atomic model. The so-calculated intensities are then compared with the measured intensities, and the best model is the one that minimizes $M$ :

$$
M=\sum w\left(F_{\mathrm{o}}^{2}-F_{\mathrm{c}}^{2}\right)^{2} .
$$

In this equation $w$ is a weight applied to every structure factor expressing our confidence in the corresponding observation (e.g. $w=1 / \sigma$, where $\sigma$ is the standard uncertainty of $F_{\mathrm{o}}^{2}$ ), $F_{\mathrm{o}}$ is the observed structure factor and $F_{\mathrm{c}}$ the structure factor calculated from the current model. ${ }^{6}$ In the presence of restraints the minimization function changes as follows:

$$
M=\sum w\left(F_{\mathrm{o}}^{2}-F_{\mathrm{c}}^{2}\right)^{2}+\sum 1 / \sigma^{2}\left(R_{\mathrm{t}}-R_{\mathrm{o}}\right)^{2} .
$$

In this equation, $\sigma$ is the standard uncertainty or elasticity assigned to a restraint, $R_{t}$ is the target value the restraint assigns to a quantity and $R_{\mathrm{O}}$ is the actual value of the restrained quantity as observed in the current model. A comparison of Equations (1) and (2) makes evident the equivalent role of diffraction data and restraints in the refinement.

In many refinements, restraints may not be needed at all. However, when the datato-parameter ratio is low, or when correlations among certain parameters occur (e.g. for the refinement of disorders, pseudo-symmetry or twinned structures), restraints can become essential. ${ }^{7}$ Whenever restraints are used the target values of the restraints should

\footnotetext{
${ }^{6}$ Note that this minimization function uses squared structure factors, which correspond directly to intensities. Historically, refinements were performed against $F$-values rather than $F^{2}$, however there is a number of advantages to using Equation (1) instead. Not the least of these advantages is that very weak reflections, which can sometimes be determined with negative intensities when the average noise level surpasses the signal of a very weak reflection, can be included into a refinement against $F^{2}$ values while refinement against $F$ would require the extraction of the square root of a negative number. To circumvent this problem, negative measurements must be set to zero, or to an arbitrary small positive value in a refinement against $F$. Such an approach introduces bias, as the very weak reflections do contain information and systematically ignoring them affects the structure determination. Another problem in the use of $F$-values arises from the difficulty of estimating the $\sigma(F)$ values from the $\sigma\left(F^{2}\right)$ values, the latter of which are determined during data reduction. As the least-squares method is very sensitive to the weights applied to each reflection in the above summation, problems with the $\sigma$ estimation lead to inaccuracies in the refinement. Refinement against $F^{2}$ does not cause any of these problems and even has additional advantages: It makes the refinement of twinned structures mathematically simpler, and refinement against squared structure factors is less likely to settle into a local minimum. Therefore, refinement against $F^{2}$ is superior to refinement against $F$, even though some more traditional crystallographers still insist on refining against structure factors. For a broader discussion of this matter, the interested reader may turn to the articles by Hirshfeld and Rabinovich (10) and Arnberg et al. (11).

${ }^{7} \mathrm{Keep}$ in mind that the diffraction pattern is the Fourier transformation of the spatial average over the whole crystal of the unit cell's electron density. That means, disorder refinement is based on data containing information about all components of the disorder at the same time. For the refinement program it is as if the atoms of all components were actually present simultaneously. Thus atoms from different components can partially overlap in the spatial average, which leads to sometimes strong correlation effects between the parameters of those atoms. Introducing similarity restraints on both geometry and anisotropic displacement parameters for all disordered atoms should be standard and there should be no disorder refinement without similarity restraints.
} 
be compared with those resulting from the refinement to check how the restraints are working [with SHELXL (6) a list of 'most disagreeable restraints' is found in the first file; other refinement programs like Crystals (17) offer similar lists]. A large difference between target and observed value indicates that a restraint has been overruled by the diffraction data, which means that the validity of this restraint needs to be verified carefully. If appropriate, the standard uncertainty assigned to a restraint can be decreased, thus giving the restraint a greater weight. Restraints must be applied with great care and only if justified. When appropriate however, they should be used without hesitation, and having more restraints than parameters in a refinement is nothing to be ashamed of.

In general there are two different types of restraints, direct restraints and relative restraints, and within both types one finds geometrical restraints and restraints on anisotropic displacement parameters (ADP). Direct restraints assign outside values to certain parameters of a model, while relative restraints, which are also called similarity restraints, relate equivalent parameters within a model. To include, for example, information about carbon-carbon single bonds into a refinement, one could either say 'all carbon-carbon single bonds in our model should be approximately $1.54 \AA$ long' or one could say 'all carbon-carbon single bonds in our model should have approximately the same value whatever that value may be'. The first sentence describes a direct restraint taking into account results from spectroscopy and/or other sources like databases. ${ }^{8}$ The second sentence corresponds to a similarity restraint relying only on information encoded in the diffraction data, coupled with a simple chemically sensible assumption. The value of $1.54 \AA$ is a decent target value for a $\mathrm{C}-\mathrm{C}$ bond, but using it means to rely on outside information. It is likely that the relative restraint would give a similar result - assuming the unit cell is correct and no mistakes were made during data collection and data reduction just without information from outside the current diffraction experiment. Therefore, whenever possible similarity restraints should be given preference over direct restraints. It should be pointed out that this is just an example to illustrate the difference between direct and similarity restraints, and usually one would not restrain all carbon-carbon single bonds in a model to the same target value.

The most common geometry restraints are on bond lengths, angles ${ }^{9}$ and assigning planarity to a number of atoms that are supposed to form a common plane. Typical elasticities for geometry restraints are $0.02 \AA$ for bonds, $0.04 \AA$ for 1,3 -distances (i.e. bond angles) and $0.1 \AA^{3}$ for planarity restraints.

The most important restraint on anisotropic displacement parameters is based on Hirshfeld's rigid bond theorem (19). It is applied to atoms bound to one another and restrains the anisotropic displacement parameters of two atoms in the direction of the bond between them to be equal within a given standard uncertainty (e.g. $0.01 \AA^{2}$ ). This is a physically highly sensible assumption and rigid bond restraints can be applied in many situations without too much hesitation. For example, when the data-to-parameter ratio is low (low resolution data) it is a good idea to apply rigid bond restraints to all atoms to improve the stability of the refinement.

\footnotetext{
${ }^{8}$ For example, the Cambridge Structural Database (CSD), maintained by the Cambridge Crystallographic Data Centre, contains extensive structural data of over 450,000 organic and metal-organic crystal structures (18). Based on almost a half million structures, the CSD can provide good standard values for bond lengths and angles, which can be used as targets for direct restraints.

${ }^{9}$ In SHELXL bond angles are addressed as 1,3-distances.
} 

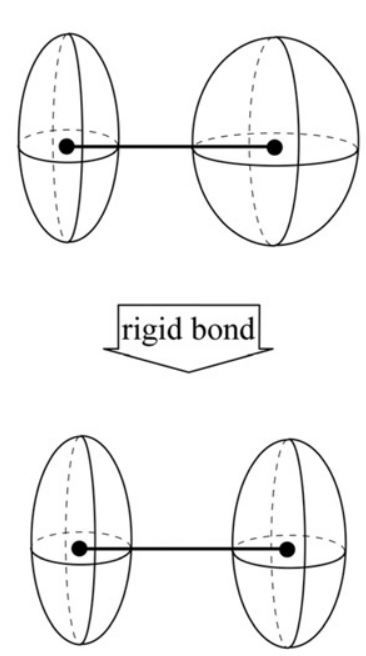
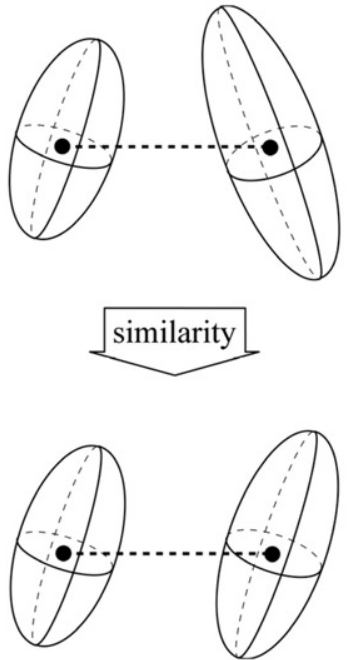
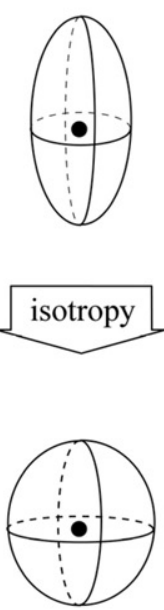

Figure 2. Effect of the three ADP restraints on anisotropic displacement parameters. The rigid bond restraint assumes that for atoms bound to one another the amplitude of motion along the direction of the bond is similar (suggested elasticity $0.01 \AA^{2}$ ). The similar ADP restraint assumes that atoms close to one another would move similarly with respect to direction and amplitude (suggested elasticity $0.04 \AA^{2} ; 0.08 \AA^{2}$ for terminal atoms). The isotropy restraint assumes that the atomic motion is approximately spherical (suggested elasticity $0.1 \AA^{2} ; 0.2 \AA^{2}$ for terminal atoms). Figure modified from (1).

Another sensible assumption, albeit of somewhat less general applicability, is that atoms which are close to one another would move in similar directions with approximately similar amplitudes (20). Thus, atoms closer to one another than say $1.7 \AA$ can be restrained to have the same $U^{i j}$ components within a standard uncertainty of, for example, $0.04 \AA^{2}$. This assumption is somewhat bolder than the Hirshfeld theorem; hence the fourfold larger standard uncertainty, which gives the restraint a much weaker weight. For terminal atoms an even larger value for the standard uncertainty is appropriate (say $0.08 \AA^{2}$ ), and groups that can rotate freely, such as $\mathrm{CF}_{3}$ or sulfate ions, should not be subjected to the similar ADP restraint at all. Note that rigid bond restraints, which are only effective in the direction of the bond, are still applicable to rotating groups.

A third commonly used ADP restraint assumes approximate isotropic behaviour of otherwise anisotropically refined atoms. This assumption should only be applied as an option of last resort and the use of a comparatively high standard uncertainty is important (e.g. $0.1 \AA^{2}$ ). A typical situation for this restraint is for free water molecules in large organic or biological structures. This restraint is easily abused and must not be mistaken for a convenient way to make anisotropic displacement ellipsoids look better. Pathological ellipsoids should always be investigated and not massaged away with restraints. Figure 2 [modified from (1)] depicts the effect of the three ADP restrains.

In addition to geometry and ADP restraints, there are several others: Shift-limiting restraints can be applied to refinements that do not converge well and limit the amount a parameter can change during each refinement cycle. Anti-bumping restraints apply a repulsive force to atoms that approach one another closer than the sum of their radii 
plus a specified distance. Floating origin restraints keep refinements in polar space groups ${ }^{10}$ stable by restraining the sum of all parameters to be constant. This method was introduced by Flack and Schwarzenbach (21) and is more stable and flexible than constraining the polar coordinate(s) of one atom to be zero; a low elasticity for the floating origin restraint makes it almost equivalent to a constraint.

\subsection{General strategies}

It is always good to advance slowly with difficult structures. This can start as early as picking the first atoms from the list of electron density maxima. When in doubt about a specific peak, do not include it into the model. If the peak is in fact an atom it will reappear as part of the next difference Fourier map; if it is not, including it may introduce a bias, making it more difficult to advance with the model building. With difficult structures, it can take many refinement cycles to find all non-hydrogen atoms and taking it step-by-step is the only way.

When refining disorder, first model the non-hydrogen atoms while keeping them isotropic. Once the refinement is stable, allow for anisotropic refinement and then, finally, add the hydrogen atoms. If there are several disorders in a structure, refine them one at a time and if a disorder involves many atoms (say more than $20 \%$ of all independent atoms), refine it in portions. Always remember, you are dealing with a Fourier synthesis and every atom contributes to the phase of every structure factor. In turn, every structure factor with its phase contributes to the electron density function as a whole and hence influences every individual atom. That means in crystal structure refinement everything influences everything and if you start too many construction sites at the same time, the refinement may become unstable.

For the same reason that, through the Fourier relationship between coordinates, phases and electron density, every single atom contributes to all atoms in the structure, it is very important not to neglect the refinement of solvent molecules. On average a crystallographer spends more time modelling solvent atoms and counter ions than target molecules, but taking into account that the main molecule can be described more accurately when the solvent model is sound, this is time well spent.

Always apply restraints as you go. It is a common mistake to say 'I will split those atom sites into two components now and apply the similarity restraints later when I see how it goes'. Odds are it will not go well unless the restraints are applied right away (see footnote 7). It can even be required to apply stricter restraints at first and relax or release them later on when the refinement has stabilized.

It is good practice to always introduce a sensible naming scheme for all atoms and to bring them into a logical order within the input file before doing anything else. It should be your goal to be able to tell simply from the name of an atom where in the model the atom sits, which atoms are its neighbours and which other atoms in your model share the same geometry. This will help with the parameterization of disorders and the application of similarity restraints and, depending on the refinement program, may facilitate a number of additional tasks, such as hydrogen atom generation.

\footnotetext{
${ }^{10}$ For example, $P 1$ where the origin is entirely arbitrary, or $P 2_{1}$ where the origin can be anywhere on the $b$ axis.
} 
When dealing with a twinned structure, include the twin law as early as possible. Once the twin law is found, and programs like TwinRotMat in PLATON (22) or Rotax (23) make this really easy, there is no reason not to include it into the refinement right away. Twinning still has the stigma of being difficult; however a twinned structure is much easier to refine than a disordered one, as all you need is the twin law and you are good to go. When a structure appears to be difficult and shows some twin-warning signs [see (3) or Chapter 7 in (1)], it is comparatively easy to try twinning first, before spending hours or days on refining disorders or pseudo-symmetry.

Check bond lengths and angles for sensibility. If all bonds are systematically too long or too short, it is likely that there is a problem with the data or the unit cell. If many bonds deviate significantly from expected values, some too long, others too short, check whether the space group is correct (an overlooked inversion centre can sometimes lead to dramatic distortions, as demonstrated in Section 4.4). If only a few bonds are longer or shorter than expected, check for errors in the assignment of atom types or look into disorders (such a situation is described in Section 4.3).

Always look at a plot of the anisotropic displacement ellipsoids [commonly called 'Ortep plot', referring to the program ORTEP by Johnson (24)]. The shape and size of the thermal ellipsoids is one of the most important indicators for problems with the molecular model. A very small ellipsoid indicates that the corresponding atom may in fact be heavier than the one currently contained in the model (e.g. oxygen refined as carbon), a very large ellipsoid could mean the opposite or indicate disorder. Elongated, cigar shaped ellipsoids are another sign indicating disorder. Flat, pancake shaped ellipsoids usually point out problems with pseudo-symmetry or incorrect space groups.

Use automated validation software such as PLATON (22) before publishing a structure. Everybody makes mistakes and automated validation programs point out potential problems that should be looked into.

\section{Examples}

As seen above, there are only three things a crystallographer needs: basic understanding of diffraction theory and general chemistry, patience, and practice in the use of constraints and restraints. While the first requirement is beyond the scope of this article, the following examples will demonstrate how the second and third come into play in the everyday life of a crystallographer. It is no coincidence that the majority of the examples below describe disorders, as disordered molecules are by far the most labour intense aspect of structure refinement.

\subsection{Splitting coordinates step-by-step}

Disorders sometimes reveal themselves only atom-by-atom. Figure 3(a) shows a complete anisotropic model of a molecule containing a conjugated aromatic system with two $n$-hexyl groups. While one of them, $\mathrm{C}(801)$ to $\mathrm{C}(806)$, seems to be well-behaved, the thermal ellipsoids of atoms $\mathrm{C}(105)$ and $\mathrm{C}(106)$ are clearly elongated, suggesting a disorder of the last two atoms of this hexyl chain. Additional evidence for this assumption is provided by two of the four highest residual density maxima, which are found in locations suitable for the second positions of atoms $\mathrm{C}(105)$ and $\mathrm{C}(106)$. Splitting the coordinates of those two atoms, using $\mathrm{Q}(2)$ and $\mathrm{Q}(4)$ as initial positions for $\mathrm{C}(06 \mathrm{~A})$ and $\mathrm{C}(05 \mathrm{~A})$, respectively, and 

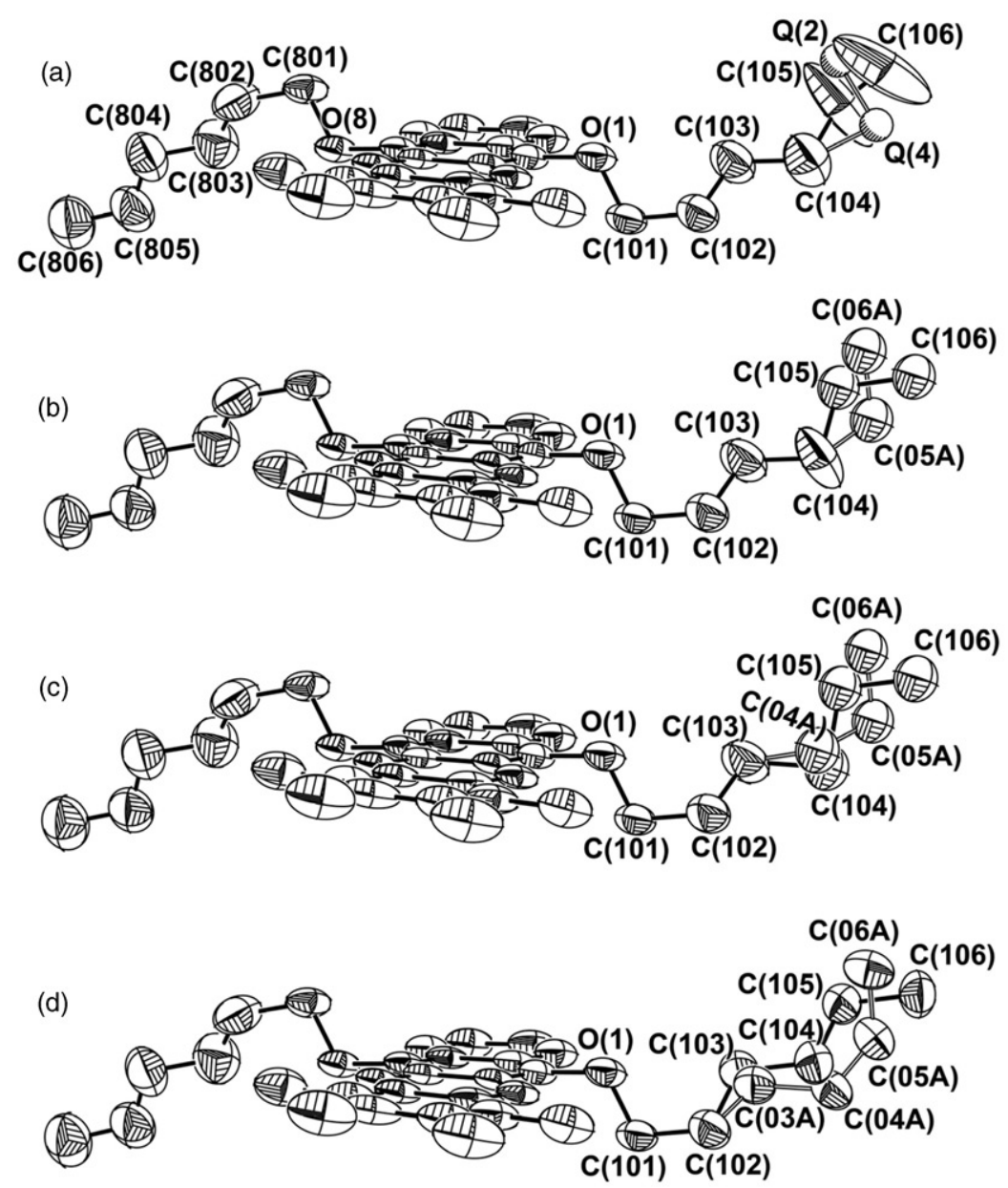

Figure 3. Step-by-step refinement of a disordered $n$-hexyl chain. (a) Initial model with elongated thermal ellipsoids for atoms $\mathrm{C}(105)$ and $\mathrm{C}(106)$; residual electron density maxima $\mathrm{Q}(2)$ and $\mathrm{Q}(4)$ are located at the alternative sites for these two atoms. (b) Disorder of C(105) and C(106) is modelled (disordered atoms isotropic), the thermal ellipsoid of C(104) is now elongated. (c) Disorder for atom $\mathrm{C}(104)$ included as well (all disordered atoms still isotropic), the thermal ellipsoid of C(103) is now slightly elongated. (d) Disorder refined for the four terminal carbons, all atoms anisotropic; thermal ellipsoid for C(102) looks good, hence the disorder refinement of the $n$-hexyl chain is complete. All thermal ellipsoids are drawn at the $50 \%$ probability level; all hydrogen atoms omitted for clarity.

refining them as two components of a disorder (at first isotropic, of course) gives rise to the model shown in Figure 3(b). Similarity restraints were applied to make the bond lengths and angles of both components of the disorder equivalent to one another and as well to the not disordered $n$-hexyl chain. ${ }^{11}$ Similar ADP restraints were applied to the four

\footnotetext{
${ }^{11}$ It is frequently helpful to relate disordered groups to chemically identical well-behaved groups by means of geometrical similarity restraints. In this case we have a disordered $n$-hexyl chain and a not disordered one; it makes sense and stabilizes the refinement to restrain the corresponding bond lengths and angles to be equivalent.
} 
disordered atoms. Rigid bond restraints are not applicable to isotropic atoms and were not introduced at this time. Table 1 gives an explicit summary of all restraints used during the refinement of this disorder.

The two disordered atoms in the current model are well behaved, however the next carbon, $\mathrm{C}(104)$, now seems to be disordered too, as its thermal ellipsoid is somewhat elongated [Figure 3(b)]. This was not visible before, but modelling the disorder of the first two carbon atoms improved the model enough to make further disorder appear. After modelling the disorder of $\mathrm{C}(104)$ - that means splitting the position and introducing more similarity restraints - yet one more atom, C(103), appears to be involved in the disorder, as can be seen in Figure 3(c). After refining all disordered atoms, first isotropically and then anisotropically, hydrogen atoms can be added. Note that while carbon atom $\mathrm{C}(102)$ is not disordered in its position, the hydrogen atoms on it are and ignoring this fact is a common mistake. The final model is shown in Figure 3(d). This kind of successive modelling of disorders is typically necessary when, as is the case here, the two components are not very different from one another (relatively small distances between disorder-related atoms) and/or when the difference in occupancy between the components is relatively large. If the two components are very close to one another (distances of, say, $0.3 \AA$ between disorder related pairs of atoms) or if the minor component has a barely significant occupancy [e.g. the ratio refines to $0.95(2)$, which corresponds to an occupancy of the minor component of only $5 \%$ within 2 percentage points], it may be just as well to ignore the disorder altogether and somewhat larger thermal ellipsoids may describe the situation better. Then again: $5 \%$ of a tungsten atom is still equivalent to almost four electrons, which may very well be worth modelling. Below, an example will be given (Section 4.3) where refining a 92:8 disorder makes the difference between an unreasonable model and a good structure. As frequently in life and in crystallography, it depends on the situation and there is no substitute for experience.

\subsection{Disordered $\mathrm{CF}_{3}$ groups}

Two of the most disorder prone chemical groups are probably tert-butyl $\left({ }^{t} \mathrm{Bu}\right)$ and trifluoro-methyl $\left(\mathrm{CF}_{3}\right)$. Their high symmetry, which frequently makes them fit into the crystal packing in many orientations, coupled with the relatively low energy barrier for rotation about the threefold symmetry axis is the reason for many of these groups to be found disordered over at least two positions. Figure 4(a) shows a disordered $\mathrm{CF}_{3}$ group where the disorder has not yet been modelled. Even if one assumes more than two positions or even free rotation, modelling of two components as shown in Figure 4(b) and (c) is usually sufficient. A freely rotating $\mathrm{CF}_{3}$ group can be represented adequately by six fluorine positions with elongated thermal ellipsoids [Figure 4(c)]. The following constraints and restraints should, can or might be applied to disordered $\mathrm{CF}_{3}$ (the same restraints apply to ${ }^{t} \mathrm{Bu}$ groups, only the atom names and types are different).

All six C-F distances should be restrained to be equal (suggested elasticity $0.02 \AA$ ), thus making the distances between the following atom pairs equivalent: $(\mathrm{C} 1)-\mathrm{F}(1), \mathrm{C}(1)-\mathrm{F}(2)$, $\mathrm{C}(1)-\mathrm{F}(3), \quad(\mathrm{C} 1)-\mathrm{F}(1 \mathrm{~A}), \quad \mathrm{C}(1)-\mathrm{F}(2 \mathrm{~A}), \quad \mathrm{C}(1)-\mathrm{F}(3 \mathrm{~A}), \quad$ assuming the atomic labelling scheme shown in Figure 4. All six F..F distances (which correspond to the $\mathrm{F}-\mathrm{C}-\mathrm{F}$ angles) should be restrained to be equal (suggested elasticity $0.04 \AA$ ), making the distances between the following atom pairs equivalent: $F(1) \cdots F(2), F(2) \cdots F(3)$, $\mathrm{F}(3) \cdots \mathrm{F}(1), \mathrm{F}(1 \mathrm{~A}) \cdots \mathrm{F}(2 \mathrm{~A}), \mathrm{F}(2 \mathrm{~A}) \cdots \mathrm{F}(3 \mathrm{~A}), \mathrm{F}(3 \mathrm{~A}) \cdots \mathrm{F}(1 \mathrm{~A})$. Assuming the atom to 
Table 1. Similarity restraints as applied for the step-by-step refinement of a disorder of the last four atoms of an $n$-hexyl chain.

\begin{tabular}{|c|c|c|c|c|}
\hline Step & Restraint type & Elasticity & $\begin{array}{l}\text { Distances/atoms to } \\
\text { which the restraints are applied }\end{array}$ & No. \\
\hline \multirow[t]{4}{*}{1} & Similar distance & $0.02 \AA$ & $\mathrm{C}(104)-\mathrm{C}(105) \approx \mathrm{C}(104)-\mathrm{C}(05 \mathrm{~A})$ & 10 \\
\hline & & & $\mathrm{C}(105)-\mathrm{C}(106) \approx \mathrm{C}(05 \mathrm{~A})-\mathrm{C}(06 \mathrm{~A})$ & \\
\hline & Similar distance & $0.04 \mathrm{~A}$ & $C(103) \cdots C(105) \approx C(103) \cdots C(05 A)$ & \\
\hline & Similar ADP & $0.04 \AA^{2}$ & $\begin{array}{l}\mathrm{C}(104) \cdots \mathrm{C}(106) \approx \mathrm{C}(04 \mathrm{~A}) \cdots \mathrm{C}(06 \mathrm{~A}) \\
\mathrm{C}(105), \mathrm{C}(106), \mathrm{C}(05 \mathrm{~A}), \mathrm{C}(06 \mathrm{~A})\end{array}$ & \\
\hline \multirow[t]{7}{*}{2} & Similar distance & $0.02 \AA$ & $\mathrm{C}(103)-\mathrm{C}(104) \approx \mathrm{C}(103)-\mathrm{C}(04 \mathrm{~A})$ & 17 \\
\hline & & & $\mathrm{C}(104)-\mathrm{C}(105) \approx \mathrm{C}(04 \mathrm{~A})-\mathrm{C}(05 \mathrm{~A})$ & \\
\hline & & & $\mathrm{C}(105)-\mathrm{C}(106) \approx \mathrm{C}(05 \mathrm{~A})-\mathrm{C}(06 \mathrm{~A})$ & \\
\hline & Similar distance & $0.04 \AA$ & $\mathrm{C}(102) \cdots \mathrm{C}(104) \approx \mathrm{C}(102) \cdots \mathrm{C}(04 \mathrm{~A})$ & \\
\hline & & & $\mathrm{C}(103) \cdots \mathrm{C}(105) \approx \mathrm{C}(103) \cdots \mathrm{C}(05 \mathrm{~A})$ & \\
\hline & & & $\mathrm{C}(104) \cdots \mathrm{C}(106) \approx \mathrm{C}(04 \mathrm{~A}) \cdots \mathrm{C}(06 \mathrm{~A})$ & \\
\hline & Similar ADP & $0.04 \AA^{2}$ & $\begin{array}{l}C(105), C(106), C(05 A), C(06 A), \\
\quad C(104), C(04 A)\end{array}$ & \\
\hline \multirow[t]{9}{*}{3} & Similar distance & $0.02 \AA$ & $\mathrm{C}(102)-\mathrm{C}(103) \approx \mathrm{C}(102)-\mathrm{C}(03 \mathrm{~A})$ & 22 \\
\hline & & & $\mathrm{C}(103)-\mathrm{C}(104) \approx \mathrm{C}(03 \mathrm{~A})-\mathrm{C}(04 \mathrm{~A})$ & \\
\hline & & & $\mathrm{C}(104)-\mathrm{C}(105) \approx \mathrm{C}(04 \mathrm{~A})-\mathrm{C}(05 \mathrm{~A})$ & \\
\hline & & & $\mathrm{C}(105)-\mathrm{C}(106) \approx \mathrm{C}(05 \mathrm{~A})-\mathrm{C}(06 \mathrm{~A})$ & \\
\hline & & & $C(101) \cdots C(103) \approx C(101) \cdots C(03 A)$ & \\
\hline & Similar distance & $0.04 \AA$ & $\mathrm{C}(102) \cdots \mathrm{C}(104) \approx \mathrm{C}(102) \cdots \mathrm{C}(04 \mathrm{~A})$ & \\
\hline & & & $\mathrm{C}(103) \cdots \mathrm{C}(105) \approx \mathrm{C}(03 \mathrm{~A}) \cdots \mathrm{C}(05 \mathrm{~A})$ & \\
\hline & & & $\mathrm{C}(104) \cdots \mathrm{C}(106) \approx \mathrm{C}(04 \mathrm{~A}) \cdots \mathrm{C}(06 \mathrm{~A})$ & \\
\hline & Similar ADP & $0.04 \AA^{2}$ & $\begin{array}{l}\mathrm{C}(103), \mathrm{C}(104), \mathrm{C}(105), \mathrm{C}(106), \mathrm{C}(03 \mathrm{~A}), \\
\mathrm{C}(04 \mathrm{~A}), \mathrm{C}(05 \mathrm{~A}), \mathrm{C}(06 \mathrm{~A})\end{array}$ & \\
\hline \multirow[t]{10}{*}{4} & Similar distance & $0.02 \AA$ & $\mathrm{C}(102)-\mathrm{C}(103) \approx \mathrm{C}(102)-\mathrm{C}(03 \mathrm{~A})$ & 102 \\
\hline & & & $\mathrm{C}(103)-\mathrm{C}(104) \approx \mathrm{C}(03 \mathrm{~A})-\mathrm{C}(04 \mathrm{~A})$ & \\
\hline & & & $\mathrm{C}(104)-\mathrm{C}(105) \approx \mathrm{C}(04 \mathrm{~A})-\mathrm{C}(05 \mathrm{~A})$ & \\
\hline & & & $\mathrm{C}(105)-\mathrm{C}(106) \approx \mathrm{C}(05 \mathrm{~A})-\mathrm{C}(06 \mathrm{~A})$ & \\
\hline & Similar distance & $0.04 \AA$ & $C(101) \cdots C(103) \approx C(101) \cdots C(03 \mathrm{~A})$ & \\
\hline & & & $C(102) \cdots C(104) \approx C(102) \cdots C(04 A)$ & \\
\hline & & & $\mathrm{C}(103) \cdots \mathrm{C}(105) \approx \mathrm{C}(03 \mathrm{~A}) \cdots \mathrm{C}(05 \mathrm{~A})$ & \\
\hline & & & $\mathrm{C}(104) \cdots \mathrm{C}(106) \approx \mathrm{C}(04 \mathrm{~A}) \cdots \mathrm{C}(06 \mathrm{~A})$ & \\
\hline & Similar ADP & $0.04 \AA^{2}$ & $\mathrm{C}(103), \mathrm{C}(104), \mathrm{C}(105), \mathrm{C}(106), \mathrm{C}(03 \mathrm{~A})$, & \\
\hline & Rigid bond & $0.01 \AA^{2}$ & $\begin{array}{l}\mathrm{C}(04 \mathrm{~A}), \mathrm{C}(05 \mathrm{~A}), \mathrm{C}(06 \mathrm{~A}) \\
\mathrm{C}(103), \mathrm{C}(104), \mathrm{C}(105), \mathrm{C}(106), \mathrm{C}(03 \mathrm{~A}), \mathrm{C}(04 \mathrm{~A}), \\
\mathrm{C}(05 \mathrm{~A}), \mathrm{C}(06 \mathrm{~A})\end{array}$ & \\
\hline
\end{tabular}

Note: The fourth column gives the total number of similarity restraints applied to the hexyl chain at each step. Step 1: A disorder model is refined for the last two atoms of the hexyl chain, the disordered atoms are isotropic. Step 2: Disorder refined for the last three atoms, the disordered atoms are isotropic. Step 3: Disorder refined for the last four atoms of the hexyl chain, the disordered atoms are isotropic. Step 4: No additional disorder refined, all atoms anisotropic. The 19 geometry restraints ( 10 distances plus 9 angles) relating the partially disordered $n$-hexyl chain to the well-behaved one, are not included in the table. Note that the number of similar ADP restraints depends on the proximity of the disordered atoms to one another and also to other atoms within the model and is hence a function of not only the number of atoms the restraints are applied to. 

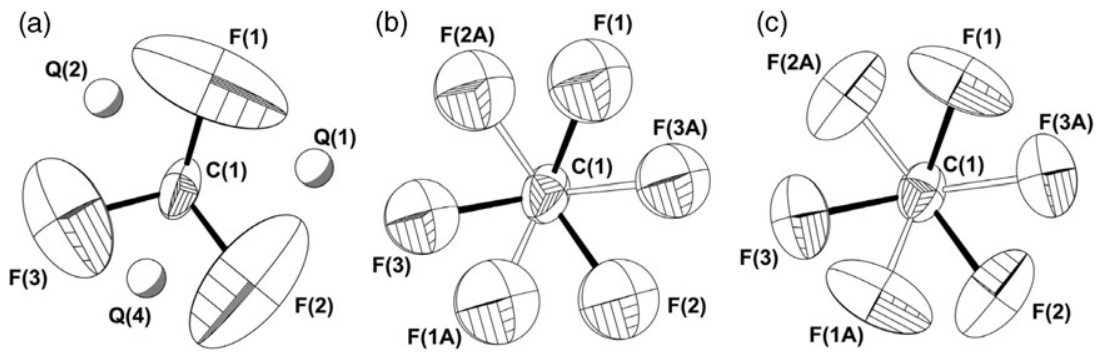

Figure 4. 50 percent thermal ellipsoid drawing of a disordered trifluoro-methyl group. (a) Before modelling the disorder, the three residual density maxima $\mathrm{Q}(1), \mathrm{Q}(2)$ and $\mathrm{Q}(4)$ correspond to the fluorine atoms of the second component of the disorder. (b) Disorder modelled over two positions, fluorine atoms still isotropic, (c) same as (b) but fluorine atoms anisotropic. The atom to which the $\mathrm{CF}_{3}$ group binds, in the text referred to as $\mathrm{X}$, is hidden behind atom $\mathrm{C}(1)$ in the figure.

which the $\mathrm{CF}_{3}$ group connects is called $\mathrm{X}$, all six $\mathrm{F} \cdots \mathrm{X}$ distances (which correspond to the $\mathrm{F}-\mathrm{C}-\mathrm{X}$ angles) should be restrained to be equal (suggested elasticity $0.04 \AA$ ) to keep the $\mathrm{CF}_{3}$ group from tilting relative to the $\mathrm{X}-\mathrm{C}$ bond; this makes the distances between the following atoms pairs equivalent: $X \cdots F(1), \quad X \cdots F(2), \quad X \cdots F(3), \quad X \cdots F(1 A)$, $\mathrm{X} \cdots \mathrm{F}(2 \mathrm{~A}), \mathrm{X} \cdots \mathrm{F}(3 \mathrm{~A})$. In addition, rigid bond restraints should be applied to all six $\mathrm{F}$ atoms, and the $\mathrm{C}$ atom (suggested elasticity $0.01 \AA^{2}$ ). These 57 restraints (3 times 15 distance restraints plus 12 rigid-bond restraints $)^{12}$ should always be applied to all disordered $\mathrm{CF}_{3}$ groups.

If the atoms of the second component of the disorder tend to approach the positions of the atoms of the first component, but the thermal ellipsoids are relatively long, suggesting almost free rotation, then the six inter-component $\mathrm{F}$... F distances can be restrained to be equal (suggested elasticity at least $0.04 \AA$ ), thus making the distances between the following atom pairs equivalent: $F(1) \cdots F(3 A), F(3 A) \cdots F(2), F(2) \cdots F(1 A), \quad F(1 A) \cdots F(3)$, $\mathrm{F}(3) \cdots \mathrm{F}(2 \mathrm{~A}), \mathrm{F}(2 \mathrm{~A}) \cdots \mathrm{F}(1)$ (see Figure 4 for labelling scheme). If the thermal ellipsoids are overly elongated, isotropy restraints can be applied (suggested elasticity $0.1 \AA^{2}$ ). If there are several $\mathrm{CF}_{3}$ groups in the structure, they can be made geometrically equivalent; this works well especially if some $\mathrm{CF}_{3}$ groups are disordered while others are not (suggested elasticities $0.02 \AA$ for bonds, $0.04 \AA$ for angles).

If in spite of all the should-be-used and can-be-used restraints a disordered $\mathrm{CF}_{3}$ group still misbehaves, constraints may be introduced. It is a fair assumption that fluorine atoms on opposite sides of the disordered $\mathrm{CF}_{3}$ arrangement would show very similar anisotropic displacement parameters, both in shape and direction, as they are related by a pseudotwofold axis along the $\mathrm{X}-\mathrm{C}(1)$ bond. Therefore, the $U^{i j}$ values of the atoms $\mathrm{F}(1)$ and $\mathrm{F}(1 \mathrm{~A})$ may be constrained to be identical and similarly the ADPs of the other two atom pairs, $\mathrm{F}(2) / \mathrm{F}(2 \mathrm{~A})$ and $\mathrm{F}(3) / \mathrm{F}(3 \mathrm{~A})$ (assuming an atomic naming scheme as in Figure 4). As an extreme measure, all six fluorine atoms might be refined as a rigid group assuming perfect tetrahedral geometry within each component and the two positions rotated from each other by $60^{\circ}$.

\footnotetext{
${ }^{12}$ Note that restraining six interatomic distances to be equal introduces 15 similarity restraints, as each distance is made equivalent to all others.
} 

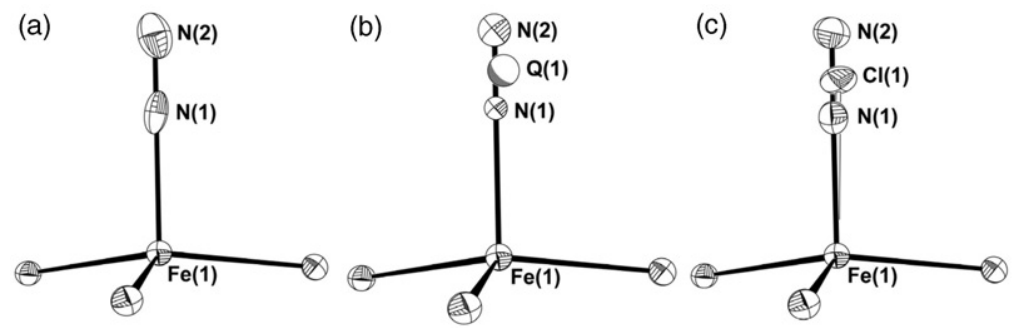

Figure 5. The 50\% thermal ellipsoid drawing of a mixed crystal refined as a disorder (only a fragment of the molecule is drawn for clarity). (a) Before modelling of the disorder the thermal ellipsoids of the nitrogen atoms are elongated and the $\mathrm{N}-\mathrm{N}$ distance is determined too short. (b) Nitrogen atoms isotropic and occupancy of nitrogen atoms reduced to $80 \%$ to make residual density peak Q(1) appear. (c) $\mathrm{Q}(1)$ interpreted as chlorine atom, which is disordered versus the $\mathrm{N}_{2}$ molecule; all atoms anisotropic, the ratio between $\mathrm{N}_{2}$ and $\mathrm{Cl}$ refines to $92: 8$.

\subsection{Too short $N-N$ bond in dinitrogen}

A structure of a compound containing a dinitrogen molecule binding end-on to an iron complex displayed a curiously short $\mathrm{N} \equiv \mathrm{N}$ distance of $1.021 \AA$, which is even shorter than the distance in free $\mathrm{N}_{2}(1.098 \AA)$. The infrared (IR) data showed the $\mathrm{N}_{2}$ vibrational frequency at $2008 \mathrm{~cm}^{-1}$ suggesting the $\mathrm{N}-\mathrm{N}$ distance should be longer than in free $\mathrm{N}_{2}$. It is a known artefact of $\mathrm{X}$-ray structure determination that $\mathrm{N}-\mathrm{N}$ and $\mathrm{C}-\mathrm{N}$ triple bonds are determined too short, as the electron density between the atoms is higher than at the actual site of the nuclei, ${ }^{13}$ however $1.02 \AA$ is too short, even taking into account this effect. Figure 5(a) shows the situation. The anisotropic displacement ellipsoids of the two nitrogen atoms are elongated along the $\mathrm{N}-\mathrm{N}$ bond and slightly tilted, but no significant residual electron density maxima are anywhere near the iron or dinitrogen and the highest residual density maximum is only $0.4 \mathrm{e} / \AA^{3}$.

As mentioned above, when there is a problem, the best strategy is to go one step back and take things slowly. Therefore, the first move was to refine the nitrogen atoms isotropically at slightly reduced occupancy ( 0.8 instead of 1$)$ hoping that residual density would appear after the refinement. As can be seen in Figure 5(b), this was indeed the case: the highest residual density maximum, $\mathrm{Q}(1)$, represents approximately 1.5 electrons and is located between $\mathrm{N}(1)$ and $\mathrm{N}(2)$. The distance of $\mathrm{Q}(1)$ from the iron centre is about $2.3 \AA$, typical for an iron chlorine distance. Indeed, the starting material for the dinitrogen compound had been the chloride, and it can be well imagined that the product cocrystallized with small amounts of educt. This scenario can be refined as a disorder.

For the next step, the two nitrogen atoms were assumed to be the first component of the disorder; $\mathrm{Q}(1)$ was turned into chlorine atom $\mathrm{Cl}(1)$ and assigned the second component. The ratio between the occupancies of the two components was allowed to

\footnotetext{
${ }^{13}$ This artefact is based on the circumstance that X-ray photons interact with electrons and not the nuclei. Therefore, the X-ray diffraction experiment gives rise to an electron density function and the atomic positions are only accurate in as much as they coincide with the electron density maxima. As an alternative method, neutron diffraction gives access to the positions of the nuclei themselves and the artefact of apparently shortened triple bonds between light atoms does not arise. Similarly, neutron diffraction is a good way to accurately determine coordinates and anisotropic displacement parameters of hydrogen atoms, where they are of specific interest.
} 
refine freely, while the sum of both occupancies was constrained to unity. At first $\mathrm{N}(1)$, $\mathrm{N}(2)$ and $\mathrm{Cl}(1)$ were refined isotropically, then, after the model had stabilized, anisotropically. Similarity and rigid bond restraints were applied on the displacement parameters of $\mathrm{Fe}(1), \mathrm{N}(1), \mathrm{N}(2)$ and $\mathrm{Cl}(1)$, but no geometry restraints were introduced. The ratio of first to second component refined to $0.915(9)$, corresponding to $92 \%$ of dinitrogen co-crystallized with $8 \%$ of starting material. The final model is shown in Figure 5(c). The final $\mathrm{N}-\mathrm{N}$ bond length is 1.118(9) $\AA$, just what one would expect, the anisotropic displacement ellipsoids look very reasonable and the residual values of the refinement improved significantly. The presence of additional electron density between the two nitrogen atoms explains both the shape of the thermal ellipsoids and the too short $\mathrm{N}-\mathrm{N}$ bond in the initial model [Figure 5(a)]. Figure 5(c) shows that the position of the chlorine atom in the final model is slightly displaced from the direct line between the two nitrogen atoms, and indeed the ellipsoids in Figure 5(a) are both directed towards the final chlorine site. Even though the occupancy of the second component in this disorder is only $8 \%$, introducing it into the model makes the difference between an unpublishable structure and an excellent one.

\subsection{Overlooked inversion centre}

As mentioned above, sometimes a structure cannot be solved in its correct centrosymmetric space group and only the expansion to a lower symmetry space group enables direct methods to solve the phase problem. The structure shown in Figure 6 could not be solved in space group $P \overline{1}$ but was solved and refined successfully in space group $P 1$ with one molecule in the asymmetric unit. At first glance, the molecule looks good and the residual values are low enough to warrant publication $[R 1=0.0636$ for $F>4 \sigma(F), w R 2=0.1862$ for all data]. When examined more carefully, however, problems become visible. The most obvious can be seen in the 'Ortep plot': The shape and direction of the thermal ellipsoids fluctuate considerably and the picture just does not look right. On closer inspection, the bond lengths and angles also fluctuate too much. The molecule has four phenyl rings, and the 24 distances between aromatic carbon atoms vary from $1.324(14) \AA$ to $1.465(12) \AA$ with an average bond distance of $1.39 \AA$. The molecule also contains two $\mathrm{AlMe}_{3}$ groups and the six Al-C distances vary from $1.929(12) \AA$ to $2.006(10) \AA$ with an average value of $1.97 \AA$. This is typical for an overlooked inversion centre, as bond lengths and other parameters that should be identical if the structure was refined in the centrosymmetric space group will be highly correlated. In technical terms: because of the nearly singular least-squares normal matrix, only the average value of a pair of bond lengths related by the overlooked inversion must be correct, the two individual values do not matter much in this situation. Not only are the fluctuations in equivalent bond distances intolerably large, the standard uncertainties of the bond lengths are also quite high. Interestingly, and quite typically for the problem at hand, the average values of the bond lengths are fine.

A first approach to fix this structure could be the application of strong similarity restraints to bond lengths and angles as well as thermal ellipsoids. ${ }^{14}$ After application of

\footnotetext{
${ }^{14}$ Rather than using similarity restraints on the anisotropic displacement parameters, it should be possible to apply identical ADP constraints to atom pairs, which are related by the overlooked inversion centre (this can be a rather cumbersome enterprise for larger structures). Such an approach would correspond more closely to a refinement in the correct space group than the restrained model; however there is no substitute for choosing the correct space group.
} 


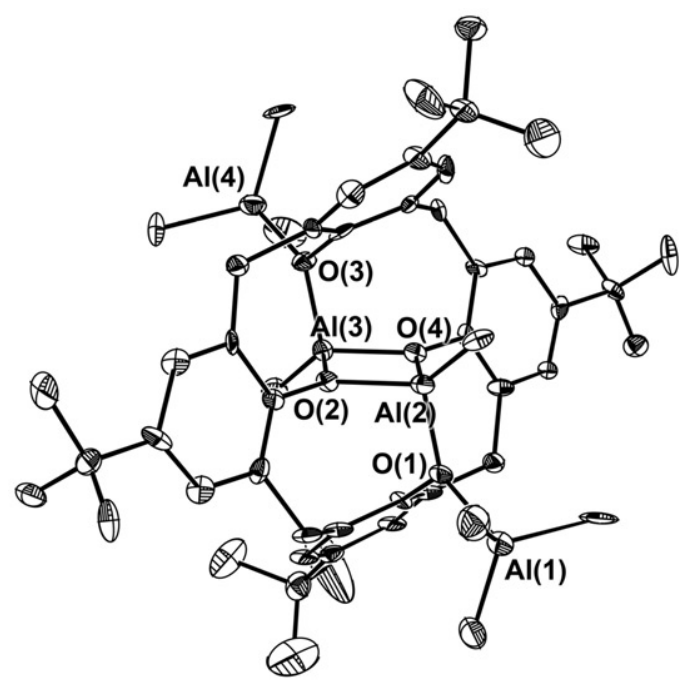

Figure 6 . The $50 \%$ thermal ellipsoid drawing of a centrosymmetric structure solved in space group $P 1$. Several thermal ellipsoids are strangely elongated or flat and the geometry is distorted. Hydrogen atoms omitted for clarity.

1537 similarity restraints with relatively small elasticities the structure looks, indeed, much better (Figure 7), even though the $R$-values are slightly worse $[R 1=0.0656$ for $F>4 \sigma(F)$, $w R 2=0.1970$ for all data]. The 24 aromatic bonds now vary from $1.385(5) \AA$ to $1.400(5) \AA$ (average value $1.39 \AA$ ) and the variation within the $\mathrm{AlMe}_{3}$ groups ranges from $1.965(5) \AA$ to $1.976(5) \AA$ with an average value of $1.97 \AA$. Note that the standard uncertainties of the bond lengths are much smaller now - this is a common effect for over-restrained structures. While the refinement with many strong restraints in space group $P 1$ works, the correct space group is still $P \overline{1}$ and the inversion centre is right in the centre of the molecule, in the middle of the $\mathrm{Al}(2)-\mathrm{O}(2)-\mathrm{Al}(3)-\mathrm{O}(4)$ four-membered ring.

Refinement in space group $P \overline{1}$ gives rise to the model shown in Figure $8 .{ }^{15}$ No restraints have been applied to the molecule at all and the variation of the bond distances ranges from $1.382(4) \AA$ to $1.398(4) \AA$ (average value $1.39 \AA$ ) for the 12 independent aromatic bonds and from $1.966(4) \AA$ to $1.975(4) \AA$ (average $1.97 \AA$ ) for the three Al-C bonds. The thermal ellipsoids as well as the standard uncertainties of the bond lengths of this completely unrestrained model in $P \overline{1}$ are even better than those of the heavily restrained model in $P 1$ and the residual values of the refinement in $P \overline{1}$ are comparable to the unrestrained $P 1$ refinement $[R 1=0.0635$ for $F>4 \sigma(F), w R 2=0.1870$ for all data]. Clearly, $P \overline{1}$ is the correct space group, even though it was necessary to solve the structure with direct methods in $P 1$. Reducing the structure to $P \overline{1}$ after solving it is not only a requirement - the structure in $P 1$ is simply wrong [see also (25)] - it is also much less work than generating 1537 restraints.

\footnotetext{
${ }^{15}$ This requires a shift of the origin to the coordinates of the inversion centre and the removal of one atom of each atom pair related by the inversion centre. In addition, the input file(s) for the refinement program need to be altered to reflect the changed symmetry. The introduction of the inversion centre cuts the asymmetric unit in half; it now contains only half a molecule.
} 


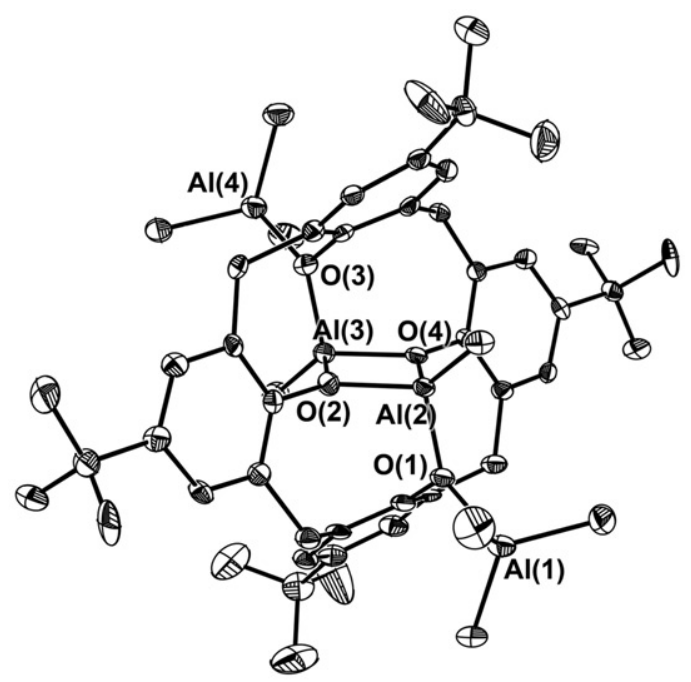

Figure 7. Same structure in as in Figure 6, still in space group P1. Application of 1537 restraints reduced the distortion of the molecule and made the thermal ellipsoids look more reasonable. Hydrogen atoms omitted for clarity.

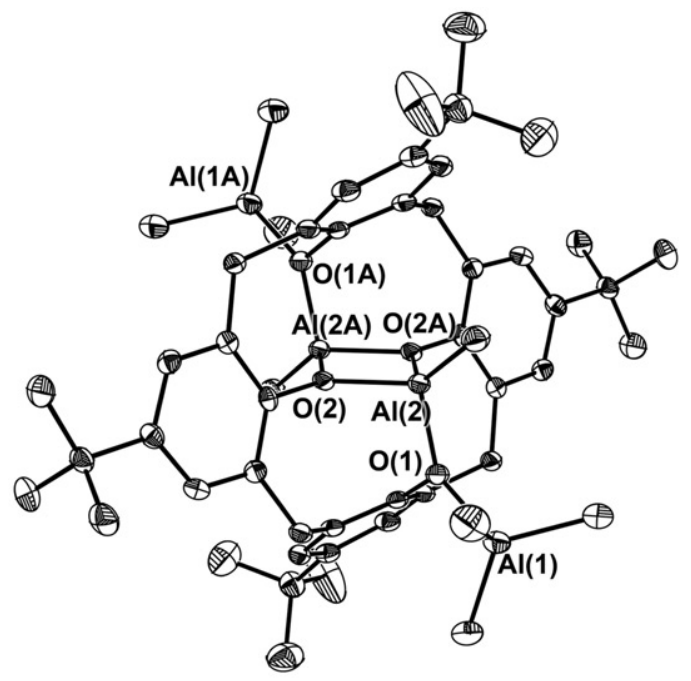

Figure 8. Same structure as in Figures 6 and 7, but now correctly refined in space group $P \overline{1}$. No restraints were applied and both geometry and thermal ellipsoids are fine. The crystallographic inversion centre is in the centre of the four-membered $\mathrm{Al}_{2} \mathrm{O}_{2}$ ring formed by $\mathrm{Al}(2), \mathrm{O}(2), \mathrm{Al}(2 \mathrm{~A})$ and $\mathrm{O}(2 \mathrm{~A})$. Hydrogen atoms omitted for clarity.

\section{Summary}

Single-crystal X-ray structure determination is one of the most powerful analytical methods and crystal structures are commonplace in most chemical journals. While there is no simple approach for the refinement of non-standard structures, this article describes and demonstrates some methods and techniques that can be used to cope with the 
challenges of crystallography. For crystal structure determination, basic chemical knowledge and an understanding of the fundamentals of crystallography is as important as patience, intuition and the skilful use of constraints and restraints. Finally, the role of experience in crystal structure determination cannot be overrated. The decision of which crystal to pick from a batch is mostly experience driven, and so to can be the choice between two space groups in the presence of pseudo-symmetry. The answer to the question of whether a specific disorder is worth refining is as much based on experience as the assessment of data quality and difference density maps. Therefore, the most important advice should be to grow crystals, to collect data and to determine many beautiful crystal structures, always critical towards the results but never losing the eye for the beauty that lies in crystallography.

\section{Notes on contributor}

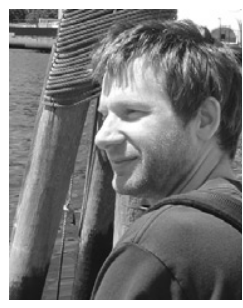

Dr. Peter Müller Director, X-Ray Diffraction Facility, Massachusetts Institute of Technology, Cambridge, MA 02139, pmueller@mit.edu.

Education: Peter Müller studied chemistry in Kaiserslautern and Göttingen, both Germany, and received his Ph.D. in Inorganic Chemistry/ Crystallography with George Sheldrick in 2001. After postdoctoral work in Molecular Biology with David Eisenberg at the University of California Los Angeles from 2001-2004 he took over as Director of the Chemistry Department's X-Ray Diffraction Facility at the Massachusetts Institute of Technology in Cambridge, MA.

Professional Activities: In addition to his duties as Director of the X-Ray Diffraction Facility and Lecturer for Crystallography at MIT Dr. Müller is a regular reviewer for the Journal of Applied Crystallography, the Journal of the American Chemical Society, the National Science Foundation and Co-Editor of Acta Crystallographica. He is Chair of the ACA Special Interest Group Service Crystallography, member of the ACA Program Committee and ACA Standing Committee Continuing Education as well as the IUCr Structural Chemistry Commission and the IUCr-Oxford University Press Book Series Committee. Dr. Müller regularly chairs sessions and workshop at international crystallographic meetings and has been teaching at all ACA Small Molecule Summer Schools since 2006. Furthermore Dr. Müller is the organizer of an annual crystallographic symposium at MIT and main Author and editor of the book Crystal Structure Refinement (IUCr Texts on Crystallography number 8, Oxford University Press 2006).

Research Interests: Data Collection Strategies, Refinement Methods, Whole Molecule Disorder, Charge Density.

\section{References}

[1] Müller, P.; Herbst-Irmer, R.; Spek, A.L.; Schneider, T.R.; Sawaya, M.R. Crystal Structure Refinement A Crystallographer's Guide to SHELXL; Oxford University Press: Oxford, UK, 2006.

[2] Clegg, W.; Blake, A.J.; Gould, R.O.; Main, P. Crystal Structure Analysis Principles and Practice; Oxford University Press: Oxford, UK, 2001.

[3] Herbst-Irmer, R.; Sheldrick, G.M. Refinement of Twinned Structures with SHELXL97. Acta Crystallogr. 1998, B54, 443-449.

[4] Herbst-Irmer, R.; Sheldrick, G.M. Refinement of Obverse/Reverse Twins. Acta Crystallogr. 2002, B58, 477-481.

[5] Watkin, D.J. The Control of Difficult Refinements. Acta Crystallogr. 1994, A50, 411-437. 
[6] Sheldrick, G.M. A Short History of SHELX. Acta Crystallogr. 2008, A64, 112-122.

[7] Watkin, D.J. Structure Refinement: Some Background Theory and Practical Strategies. J. Appl. Crystallogr. 2008, 41, 491-522.

[8] Dauter, Z. Data Collection Strategies. Acta Crystallogr. 1999, D55, 1703-1717.

[9] Müller, P.; Sawaya, M.R.; Pashkov, I.; Chan, S.; Nguyen, C.; Wu, Y.; Perry, L.J.; Eisenberg, D.S. The $1.70 \AA$ X-ray Crystal Structure of M Tuberculosis Phosphoglycerate Mutase. Acta Crystallogr. 2005, D61, 309-315.

[10] Hirshfeld, F.L.; Rabinowich, D. Treating Weak Reflexions in Least-Squares Calculations. Acta Crystallogr. 1973, A29, 510-513.

[11] Arnberg, L.; Hovmöller, S.; Westman, S. On the Significance of 'Non-Significant' Reflexions. Acta Crystallogr. 1979, A35, 497-499.

[12] Sheldrick, G.M. SADABS; University of Göttingen: Göttingen, Germany, 2008.

[13] Sheldrick, G.M. Phase Annealing in SHELX-90: Direct Methods for Larger Structures. Acta Crystallogr. 1990, A46, 467-473.

[14] Burla, M.C.; Camalli, M.; Carrozzini, B.; Cascarano, G.L.; Giacovazzo, C.; Polidori, G.; Spagna, R. SIR2002: the Program. J. Appl. Cryst. 2003, 36, 1103.

[15] Miller, R.; DeTitta, G.T.; Jones, R.; Langs, D.A.; Weeks, C.M.; Hauptman, H.A. Incorporating Tangent Refinement in the Shake-and-Bake Formalism. Science 1993, 259, 1430-1433.

[16] Flack, H.D. On Enantiomorph-Polarity Estimation. Acta Crystallogr. 1983, A39, 876-881.

[17] Betteridge, P.W.; Carruthers, J.R.; Cooper, R.I.; Prout, K.; Watkin, D.J. CRYSTALS Version 12: Software for Guided Crystal Structure Analysis. J. Appl. Crystallogr. 2003, 36, 1487.

[18] Allen, F.H. The Cambridge Structural Database: a Quarter of a Million Crystal Structures and Rising. Acta Crystallogr. 2002, B58, 380-388.

[19] Hirshfeld, F.L. Can X-ray Data Distinguish Bonding Effects from Vibrational Smearing? Acta Crystallogr. 1976, A32, 239-244.

[20] Didisheim, J.J; Schwarzenbach, D. Rigid-Link Constraints and Rigid-Body Molecules. Acta Crystallogr. 1987, A43, 226-232.

[21] Flack, H.D.; Schwarzenbach, D. On the Use of Least-Squares Restraints for Origin Fixing in Polar Space Groups. Acta Crystallogr. 1988, A44, 499-506.

[22] Spek, A.L. PLATON A Multipurpose Crystallographic Tool; Utrecht University: Utrecht, Holland, 2008.

[23] Parsons, S.; Gould, R.O. Rotax; University of Edinburgh: Edinburgh, UK, 2001.

[24] Johnson, C.K. ORTEP: A Thermal Ellipsoid Plot Program for Crystal Structure Illustrations; Oak Ridge National Laboratory: Oak Ridge, TN, 1965.

[25] Marsh, R.E. Some Thoughts on Choosing the Correct Space Group. Acta Crystallogr. 1995, B51, 897-907.

\section{Subject Index}

absorption correction 62; see also SADABS

ADP, see anisotropic displacement parameters

angles, see bond angles

anisotropic displacement parameters $63,65,70,72,74,75$

artefacts 75

atomic coordinates, see coordinates

atom type 58, 64, 70

beauty 79

binary solvent systems 72

bond angles $65,67,70,76$ 
bond lengths $65,67,70,76$

Cambridge structure database, see CSD

chemical knowledge 67, 79

chemistry, see general chemistry 70

completeness 61

constraints $64,65,70,72,74,76,79$

coordinates $58,63,65,69,70$

correlation effects 66

counter ions 69

crystal growth, see crystallization

crystal handling 60

crystallization 59, 60

Crystals 67

CSD 67

dark current 60,61

data collection strategy 61

data reduction 58, 66

detector parameters 60,61

difference Fourier synthesis 61, 69

diffraction theory 70

direct methods 62, 63, 76, 77

disagreeable restraints 67

disorder 58, 59, 62, 64, 65, 66, 69, 70-76, 79

dual space algorithm 63

elasticity, see standard uncertainty

electron density 66, 69, 70, 71, 74, 75, 76, 79

ESDs, see standard uncertainties

experience $58,61,72,79$

F2-refinement 66

F-values, see structure factors

F2-values, see structure factors

Flack parameter 64

floating origin 69

Fourier synthesis 64, 66, 69

Fourier transformation, see Fourier synthesis

fundamentals of crystallography 63, 79

general chemistry 70

guru, see local guru

high resolution limit, see resolution

Hirshfeld theorem, see rigid bond

hydrogen atoms 58, 65, 69, 72, 75

I/s 61,62

intuition 79

inversion centre $63,70,76-78$

Jedi Knight 58 
labeling scheme, see naming scheme

least squares $64,66,76$

local guru 58

low-temperature data collection 62

merging residual values 61

microscope 60

$\mathrm{MoO}$, see multiplicity of observations

mother liquor, separtion from 60

multiplicity 64

multiplicity of observations 61,62

naming scheme 69

NCS, see pseudo symmetry

non-crystallographic symmetry see pseudo symmetry

normal matrix 76

occupancy/occupancies 63, 64, 72, 75, 76

origin $63,69,77$

Ortep 70, 76; see also anisotropic displacement parameters

osf, see overall scale factor

overall scale factor 63

parameters $63,64,65,76$

patience 70,79

Patterson 62

phase angles, see phases

phases 63, 69, 76

primary beam 60,62

PLATON 70

polarizing microscope, see microscope

practice 70

pseudo-symmetry 66, 70, 74, 79

redundancy, see multiplicity of observations

refinement 58, 66, 67, 69-78

resolution $61,62,64,67$

restraints $65-69,71-74,76-79$

riding model 65

rigid bond 67, 68, 72-74, 76

rigid group constraints, see rigid group

rigid group 65,74

Roatx 70

SADABS 62

scattering factors 64

Shake-and-Bake 63

SHELXD 63

SHELXL 58, 63, 67

SHELXS 62

SIR 62

slow cooling 59 
solvent 59, 60, 65, 69

solvent systems, see binary solvent systems

space group error $62,63,70,76-78,79$

spatial average 66

special positions $64-65$

squared structure factors, see structure factors

standard uncertainties 66-69, 72-74, 76, 77

strategy, see data collection strategy

structure factors $65,66,69$

structure refinement, see refinement

structure solution 62,63

supersaturation 59,60

symmetry equivalent atoms/positions 63

symmetry equivalent reflections 61,64

test crystal 61

thermal parameters, see anisotropic displacement parameters

thermal ellipsoids 64, 68, 70, 72, 74, 76-78; see also anisotropic displacement parameters triple bonds 75

twin / twinning 59, 60, 66, 70,

TwinRotMat 70

unit cell 58,61, 66, 70

weak data 61,66

weak reflections, see weak data

wrong space group, see space group error

zero points 60 